\title{
Racism, COVID-19, and Health Inequity in the USA: a Call to Action
}

\author{
Crista E. Johnson-Agbakwu ${ }^{1,2,3,4}$ (D) Nyima S. Ali ${ }^{2,3,4} \cdot$ Corrina M. Oxford ${ }^{5} \cdot$ Shana Wingo $^{6} \cdot$ Emily Manin $^{1}$. \\ Dean V. Coonrod ${ }^{2,3,4}$
}

Received: 16 July 2020 / Revised: 27 October 2020 / Accepted: 10 November 2020 / Published online: 16 November 2020

(C) W. Montague Cobb-NMA Health Institute 2020

\begin{abstract}
The current national COVID-19 mortality rate for Black Americans is 2.1 times higher than that of Whites. In this commentary, we provide historical context on how structural racism undergirds multi-sector policies which contribute to racial health inequities such as those highlighted by the COVID-19 pandemic. We offer a concrete, actionable path forward to address structural racism and advance health equity for Black Americans through anti-racism, implicit bias, and cultural competency training; capacity building; community-based participatory research (CBPR) initiatives; validated metrics for longitudinal monitoring of efforts to address health disparities and the evaluation of those interventions; and advocacy for and empowerment of vulnerable communities. This necessitates a multi-pronged, coordinated approach led by clinicians; public health professionals; researchers; social scientists; policy-makers at all governmental levels; and local community leaders and stakeholders across the education, legal, social service, and economic sectors to proactively and systematically advance health equity for Black Americans across the USA.
\end{abstract}

Keywords COVID-19 $\cdot$ Health disparities $\cdot$ Health inequality $\cdot$ Structural racism $\cdot$ Advocacy

Structural racism, as defined by Bailey et al. is "the totality of ways in which societies foster racial discrimination through mutually reinforcing systems" which "in turn reinforce discriminatory beliefs, values, and distribution of resources." It is the underlying cause of health disparities (including COVID-19) [1]. Structural racism has its origins in the transatlantic slave trade and continues today: Black Americans are disproportionately affected by poverty, a fallible public school system, unsafe neighborhoods, food deserts, mass incarceration, police brutality, maternal and infant mortality, obesity,

Crista E. Johnson-Agbakwu

cejohn11@asu.edu

1 Southwest Interdisciplinary Research Center (SIRC), Arizona State University, Phoenix, AZ, USA

2 Obstetrics and Gynecology, Valleywise Health, University of Arizona College of Medicine, Phoenix, AZ, USA

3 Creighton University School of Medicine, Phoenix, AZ, USA

4 District Medical Group, Phoenix, AZ, USA

5 Maternal Fetal Medicine/Critical Care Medicine, NewYork-Presbyterian Hospital, Clinical Obstetrics and Gynecology - Weill Cornell Medical College, Cornell University, New York City, NY, USA

6 Arizona Oncology, Phoenix, AZ, USA and chronic health conditions, to name a few [2]. These issues have compounded synergistically in what some refer to as the COVID-19 "syndemic," but they are not new [3]. Politicians, legislators, policy-makers, economists, educators, public health professionals, and local community leaders must join in a multidisciplinary, coordinated effort to actively and systemically advance health equity. We as a nation are at an intersection in history with an obligation to take concrete steps to dismantle systemic inequities in order to improve the lives of millions of Black Americans.

The year 2020 will be punctuated in the US history as a pivotal year in which racial disparities in health were brought to the forefront and discussed in sectors outside of academic medicine and research. As of August 18, 2020, the national COVID-19 mortality rate for Black Americans was 2.1 times higher than that of Whites, and hospitalization rates for Latinos were 4.6 times than that of Whites [4]. These statistics are not new or surprising. Black women are three to four times more likely to die from pregnancy-related complications than White women and twice as likely to die from heart disease [5-7]. The prevalence of diabetes among Black Americans is 1.7 times higher than for Whites [8]. While the Centers for Disease Control and Prevention (CDC), academic institutions, and communities continue to attribute preexisting health conditions, living conditions, work-related exposures, and lack of education to the observed racial disparities in COVID-19 
infection rates and outcomes, health equity will continue to evade us until we acknowledge and address structural racism as the root cause of the problem.

The civil rights movement, which only began 66 years ago, provides perspective as to how little progress has been achieved $[9,10]$. This is evidenced by disparities in epidemics across centuries. Ideas of "black immunity" permeating the yellow fever epidemic in 1792 were perpetuated in the present pandemic [10]. The measles epidemic of 19891991 resulted in 4 to 7 fold higher rates among minority children than white children [11]. In the past 10 flu seasons, Black Americans had a flu-related hospitalization rate of 1.8 times that of White Americans [12]. These statistics are the result of policies that perpetuate structural racism and inequalities, allowing the USA's legacy of slavery to manifest in new but familiar forms. Legal segregation has transformed into redlining and de facto segregation, which, in concert with policies that uphold property taxes as a major source of funding for public school systems, reinforces disparities in quality of education. Education ultimately impacts an individual's health literacy and occupation, affecting all health outcomes [13]. In the presently struggling economy, people of color are likely to suffer more job loss as a result of the expected sequelae of the COVID-19 outbreak: $17.6 \%$ of Hispanic workers and $16.8 \%$ of Black workers work in the jobs most at risk in the current recession compared to approximately $12.5 \%$ of non-Hispanic whites [14]. A person's income and occupation determine quality of health insurance [15]. Legal segregation in hospitals has again given way to de facto segregation and discrimination by income in healthcare settings [16]. The quality and presence of insurance also impacts individuals' decisions to seek care - even in emergency situations - and, as fewer primary care providers accept Medicaid, forces them to obtain their ambulatory preventive care in the emergency department [17].

Unfortunately, Black Americans who possess quality education, good employment, and outstanding health insurance coverage still frequently receive inferior care due to implicit bias among healthcare providers [18, 19]. Regardless of income, people of color are subject to the weathering effect, which derives from chronic stress due to lifelong as well as generational exposure to discrimination and adversity (i.e., the epigenetics of slavery) [20].

At the federal level, access to equitable education and healthcare services (likely by modification of health insurance policies) is necessary. Clearly, a coordinated and multidisciplinary federal, state, and local effort is indicated. We suggest a list of concrete, actionable steps that can be taken by clinicians, public health professionals, researchers, social scientists, and policy-makers and the engagement of community stakeholders to address structural racism in advancing health equity for Black Americans (Table 1).

\section{Anti-racism, Implicit Bias, and Cultural Competency Training}

Iterative cultural competency training is fundamental to engaging all entities including local community leaders and stakeholders wherein structural racism is defined and recognized for its persistence and deep roots in the historic legacy of slavery and neocolonialism [21]. Trainings should seek to combat racism, implicit bias, and microaggressions at the institutional as well as individual level. Self-reflection exercises should examine one's own implicit biases, challenge assumptions, and consider cultural and racial power hierarchies in the patient-provider relationship; addressing the "othering" of Black bodies and the insidious ways that racial and cultural hegemony implicitly influences the patient-provider encounter [22].

Institutional policies should mandate iterative trainings across all staff and trainees that engage clinicians, public health professionals, researchers, social scientists, and policy-makers, incorporating interactive and enduring learning tools throughout the educational curricula. Trainings must also recognize the role of social determinants of health and the larger umbrella of contextualized concerns of importance to communities of color that remain contributing factors to the pervasiveness and ubiquity of health inequities.

\section{Capacity Building}

Studies indicate that communities of color receive better care when cared for by providers who share their racial and cultural identity [23]. Black Americans represent $12 \%$ of the US population yet comprise only $5 \%$ of US medical professionals [24]. To advance health equity for Black Americans, institutions must build capacity to serve communities of color by first propelling the development of pipeline training programs for healthcare professionals representing the ethno-cultural and linguistic diversity of the local communities served. Partnerships with local elementary, high schools, and colleges will facilitate longitudinal learning opportunities that exposes students to careers in the health professions and provide mentorship and financial incentives to reduce economic hardships to educational advancement. Efforts must also be extended to recruit and retain diverse faculty, staff, and trainees of color and build an infrastructure of support and mentorship to ensure their success and career advancement. We must consider the workforce integration of community health workers, cultural health navigators, and doulas into healthcare delivery systems to further nurture and sustain trust with vulnerable communities.

Efforts must ensure representation of gender and ethnocultural diversity throughout all levels of leadership within an organization. In addition, policies that affect communities 


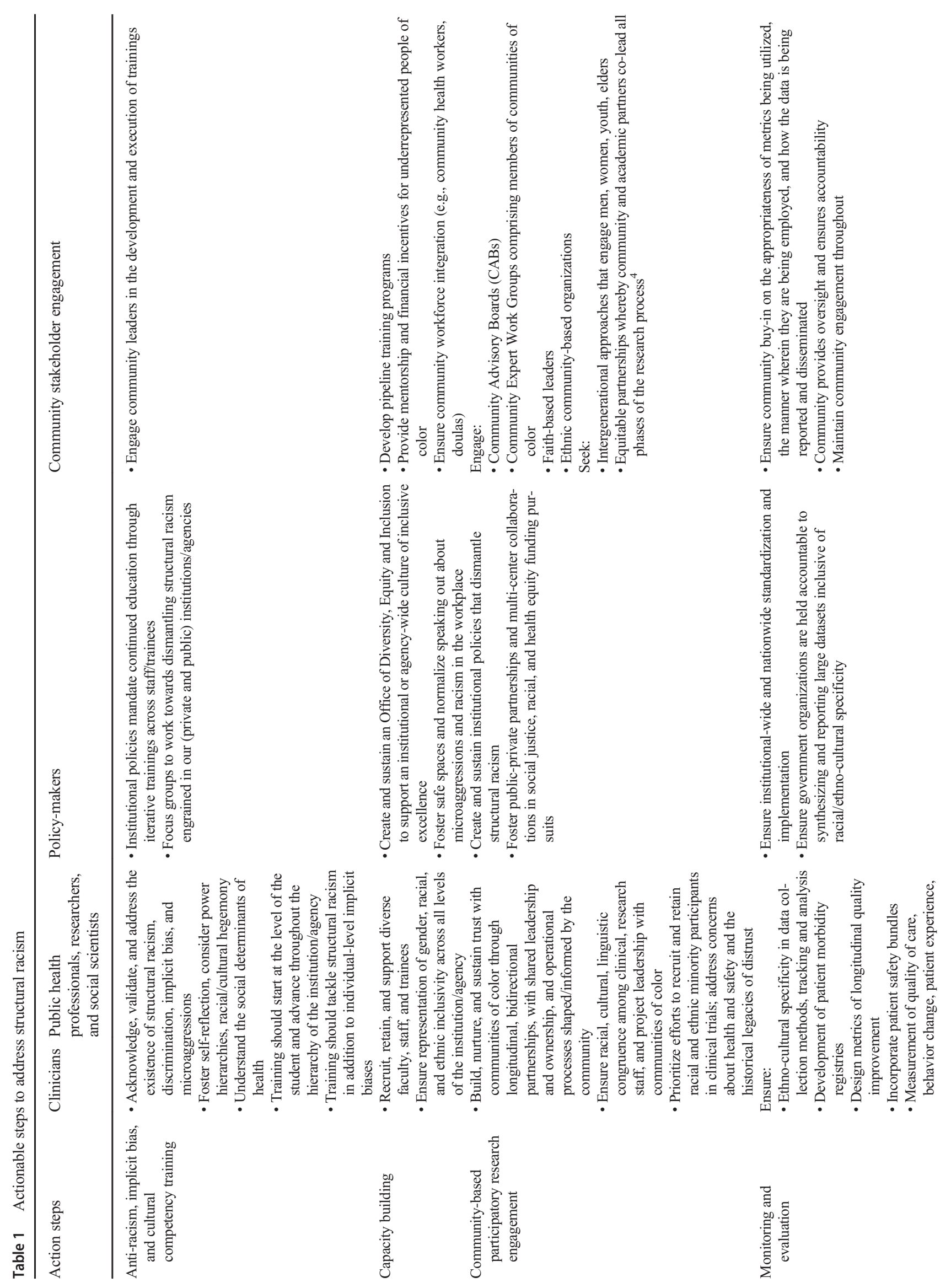




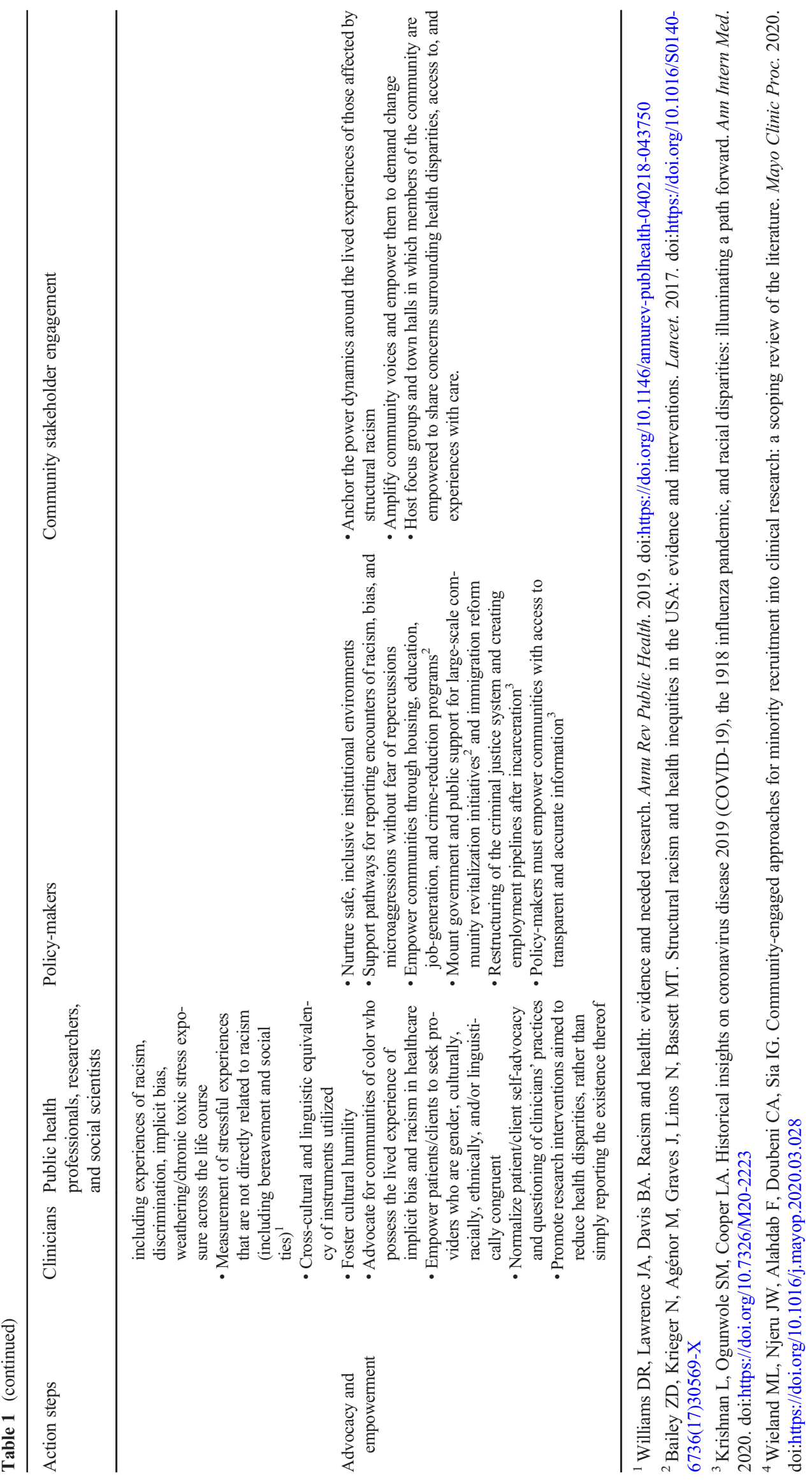


of color should include representatives from these communities in its design, implementation, interpretation, and dissemination. Institutions must establish and sustain Offices of Equity, Diversity, and Inclusion which support minority healthcare and public health trainees and professionals, as well as recognize and address their experiences of structural, institutionalized racism, discrimination, implicit bias, microaggressions, and bigotry [25]. Institutions must invest in the development of infrastructure and resources that creates safe spaces to normalize the reporting of such experiences, as well as nurture a culture of inclusive excellence.

\section{Community-Based Participatory Research Engagement}

Historical trauma from centuries of abuse, punctuated by atrocities such as the Tuskegee Syphilis Study and Henrietta Lacks' cancer cells, has generated profound distrust in the healthcare system that deters people of color from seeking treatment, confiding in their providers and complying with healthcare recommendations [26]. This distrust, in addition to factors such as financial barriers, fear of repercussions from health insurance companies, lack of access to information, and racial, cultural, and linguistic incongruence with research staff, influences minority participation in clinical trials, which further perpetuates racial disparities in health outcomes [27]. Distrust in healthcare systems and public health information may impact a community's perception of risk, leading to the propagation of unfounded myths surrounding one's own perceived susceptibility to illness [28]. Such misconceptions prevailed during the 1793 yellow fever epidemic and the beginning of the HIV epidemic and have continued during the COVID-19 pandemic $[2,29,30]$. Clinical trials must prioritize the inclusion of racial and ethnic minorities for enrollment with tangible efforts made to acknowledge and address the historic legacy of distrust and ensure racial, cultural, and linguistic congruence among clinical and research staff, as well as senior project leadership with communities of color.

We propose promoting equitable, trust-based, community partnerships that espouse the principles of community-based participatory research (CBPR) and ensure accountability by healthcare and public health institutions in equitably representing the needs, voices, and priorities of vulnerable communities through Community Advisory Boards (CABs) and/or community expert work groups [31, 32]. Educational outreach efforts that are community-centered, community-engaged, and community-led are crucial to strengthen community social networks and to build community capacity [33]. CABs empower local community champions, who are often excluded from traditional community engagement efforts, as their own community experts, to be engaged in all phases of research engagement from the initial conceptualization, design, implementation, and interpretation of the findings as well as clearly articulating and disseminating culturally and linguistically relevant findings back within their target communities.

It is also critically important to create, nurture, and sustain safe spaces where bi-directional, open dialogue can be fostered intergenerationally across men, women, youth, elders, faith-based leaders, as well as ethnic community-based organizations in order to engender mutual understanding within and across communities of color on the myriad manifestations of structural racism. Based on iterative community input, institutions must then maintain accountability for the actionable steps taken to ensure structural racism remains dismantled. Moreover, public-private partnerships and multi-center collaborations should be fostered in the pursuit of funding opportunities to advance social justice, racial, and health equity.

\section{Monitoring and Evaluation}

To actively dismantle structural racism, we must first have a strong understanding of its epidemiology and anticipate and prepare to address health inequities, rather than acknowledging them retrospectively. In the case of COVID-19, the CDC began releasing race-disaggregated data on COVID-19 mortalities nearly 5 months after the pandemic first appeared in the USA, after thousands of Americans had already died. History has repeatedly demonstrated that racial and ethnic minorities are statistically vulnerable in epidemics [10-12]; the delay in stratifying data by race and ethnicity from the outset precludes adequate preparation of public health interventions and messaging.

While current $\mathrm{CDC}$ guidelines require all laboratories analyzing COVID-19 tests to report race and ethnicity, individual states and hospitals are not mandated to report demographic data until several months after the start of the pandemic [34, 35]. Unfortunately, once these statistics are published, they are often incomplete and are allowed to include such categorizations as "unknown race" [34]. Government organizations should be held accountable to synthesize and report large population-based datasets that are inclusive of racial and ethno-cultural specificity.

Once this epidemiologic data is obtained, a multidisciplinary, coordinated approach involving cooperation among federal, legal, economic, education, public health and local community leaders to actively and systematically advance health equity is warranted. On a small scale, it is imperative that healthcare providers understand the provider-level (e.g., implicit bias), genetic, sociocultural, historical, and economic contributors to disease severity and tailor treatments and referrals accordingly. Healthcare systems and local governments must employ this race-disaggregated data, in conjunction with geospatial data, to improve access to care. These data must guide community organizations, community health workers, and social workers towards engaging the 
communities most affected by these disparities and tailoring education, interventions, advocacy programs, and public health messaging to the Black American community.

Most data on race and ethnicity coalesces distinct populations into large heterogeneous "race" categories, failing to account for the products of racism due to factors such as the "healthy immigrant effect," the "epigenetics of slavery," and the "weathering effect." [36] An important area for further research is determining the best measure that accounts for these factors and can be incorporated into large-scale epidemiological data on COVID-19 and all health measures. It is important to ensure ethno-cultural specificity in data collection methods, tracking and analysis, and achieve cross-cultural and linguistic equivalency in the instruments utilized. Patient morbidity registries should be developed, along with metrics of longitudinal quality improvement and patient safety bundles to enhance the safety and quality of clinical care. It is critical to develop and/or incorporate validated metrics to assess behavior change, quality of care, patient experience, experiences of racism, discrimination, implicit bias, the weathering effect across the life course, and exposure to chronic toxic stress. Measurement of stressful experiences unrelated to racism such as bereavement and social ties should also be considered [37].

Institutional-wide policies must ensure standardization of implementation throughout, while community stakeholders remain engaged, providing oversight and ensuring accountability as well as community buy-in on the appropriateness of the metrics being utilized, the manner in which they are being employed, and how the data is being reported.

\section{Advocacy and Empowerment}

Silence and complicity are both corrosive and destructive. It is critically important for clinicians, public health professionals, researchers, and social scientists to nurture safe environments that fosters cultural humility and embraces our collective humanity. We must be advocates for patients who possess the lived experience of implicit bias and racism within healthcare, promoting research interventions aimed to dismantle racism as part of efforts to reduce health disparities, rather than simply reporting the existence thereof. We should empower patients to seek providers who are gender, racially, ethnically, culturally, and/or linguistically congruent and normalize patient selfadvocacy in questioning clinicians' practices. One should be empowered to step outside of one's comfort zones in advocating for and speaking up for the voiceless and disempowered; speaking truth to power when encountering instances of racial prejudice, implicit bias, or microaggressions without fear of repercussions or stigmatization; and supporting pathways for reporting such encounters while nurturing safe and inclusive institutional environments.
Policy-makers should empower communities through housing, education, job generation, and crime-reduction programs and mount government and public support for largescale community revitalization initiatives and immigration reform [1]. Efforts must also be extended towards restructuring the criminal justice system and supporting employment pipelines after incarceration [10]. Moreover policy-makers must empower communities with access to transparent and accurate information [10].

Community stakeholders must have dynamics of power anchored around the lived experiences of those affected by structural racism, amplifying community voices and empowering them to demand change. Focus groups and town halls can be hosted wherein community members are empowered to share concerns encompassing health disparities, access to, and experiences with care.

Health begins far before a patient ever enters the hospital. According to the Healthy People 2020 initiative, one's health is determined in part by the social determinants of health which encompasses the environments in which people are born, live, learn, work, play, and worship [38]. All these factors contribute to the health that we, as physicians, are tasked to improve. Let us identify structural racism and barriers throughout these sectors and work within them, taking concrete, actionable steps as aforementioned, to address the root cause of health inequities. As we battle the COVID-19 pandemic, we urge clinicians, public health professionals, researchers, social scientists, policy-makers, community leaders, and scholars from across the USA to cooperate collectively, in multi-sectoral longitudinal interventions to acknowledge our history and dismantle persistent structural racism throughout the USA in ultimately ensuring sustained, long-term advancement of health equity for Black Americans [1].

Data Availability Not applicable.

\section{Compliance with Ethical Standards}

Conflict of Interest The authors declare that they have no conflict of interest.

Ethics Approval Not applicable.

Consent to Participate Not applicable.

Consent for Publication Not applicable.

Code Availability Not applicable.

\section{References}

1. Bailey ZD, Krieger N, Agénor M, Graves J, Linos N, Bassett MT. Structural racism and health inequities in the USA: evidence and 
interventions. Lancet. 2017;389:1453-63. https://oi.org/10.1016/ S0140-6736(17)30569-X.

2. Laurencin CT, McClinton A. The COVID-19 pandemic: a call to action to identify and address racial and ethnic disparities. J Racial Ethn Health Disparities. 2020;7:398-402. https://doi.org/10.1007/ s40615-020-00756-0.

3. Gravlee CC. Systemic racism, chronic health inequities, and COVID-19: a syndemic in the making? Am J Hum Biol. 2020;32:e23482. https://doi.org/10.1002/ajhb.23482.

4. COVID-19 Hospitalization and Death by Race/Ethnicity. Centers for Disease Control and Prevention.

5. National Center for Health Statistics. Health, United States Spotlight Racial and Ethnic Disparities in Heart Disease.; 2019.

6. Callaghan WM. Overview of maternal mortality in the United States. Semin Perinatol. 2012;36:2-6. https://doi.org/10.1053/j. semperi.2011.09.002.

7. Wingo SN. Black Uteri Matter. Obstet Gynecol. 2019;133:4-5. https://doi.org/10.1097/AOG.0000000000003035.

8. Ferdinand KC, Nasser SA. Racial/ethnic disparities in prevalence and care of patients with type 2 diabetes mellitus. Curr Med Res Opin. 2015;31:913-23. https://doi.org/10.1185/03007995.2015. 1029894.

9. McCoy H. Black lives matter, and yes, you are racist: the parallelism of the twentieth and twenty-first centuries. Child Adolesc Soc Work J. 2020;37:463-75. https://doi.org/10.1007/s10560-02000690-4

10. Krishnan L, Ogunwole SM, Cooper LA. Historical insights on coronavirus disease 2019 (COVID-19), the 1918 influenza pandemic, and racial disparities: illuminating a path forward. Ann Intern Med. 2020;173:474-81. https://doi.org/10.7326/M20-2223.

11. Bibbins-Domingo K. This time must be different: disparities during the COVID-19 pandemic. Ann Intern Med. 2020;173:233-4. https://doi.org/10.7326/M20-2247.

12. Centers for Disease Control and Prevention. Flu Disparities Among Racial and Ethnic Minority Groups.

13. Vable AM, Cohen AK, Leonard SA, Glymour MM, Duarte CDP, Yen IH. Do the health benefits of education vary by sociodemographic subgroup? Differential returns to education and implications for health inequities. Ann Epidemiol. 2018. https://doi. org/10.1016/j.annepidem.2018.08.014.

14. Kopf D, Detrixhe J. A coronavirus-led recession could hit jobs for young people and minorities hardest https://qz.com/1822762/ coronavirus-led-recession-in-us-could-hit-people-of-color-hardest/. Accessed April 8, 2020.

15. Song L, Wang Y, Chen B, Tan Y, Zhang W, Wang Y. The association between health insurance and all-cause, cardiovascular disease, cancer and cause-specific mortality: a prospective cohort study. Int J Environ Res Public Health. 2020;17(5):1525.

16. Niedzwiecki MJ, Hsia RY, Shen YC. Not all insurance is equal: differential treatment and health outcomes by insurance coverage among nonelderly adult patients with heart attack. J Am Heart Assoc. 2018. https://doi.org/10.1161/JAHA.117.008152.

17. Care Without Coverage.; 2002. doi:https://doi.org/10.17226/10367

18. Fiscella K, Sanders MR. Racial and ethnic disparities in the quality of health care. Annu Rev Public Health. 2016;37:375-94. https:// doi.org/10.1146/annurev-publhealth-032315-021439.

19. Understanding racial and ethnic differences in health in late life.; 2004. doi:https://doi.org/10.17226/11036

20. Mays VM, Cochran SD, Barnes NW. Race, race-based discrimination, and health outcomes among African Americans. Annu Rev Psychol. 2007;58:201-25. https://doi.org/10.1146/annurev.psych. 57.102904.190212.

21. Owens DC, Fett SM. Black maternal and infant health: historical legacies of slavery. Am J Public Health. 2019;109:1342-5. https:// doi.org/10.2105/AJPH.2019.305243.
22. Johnson-Agbakwu CE, Manin E. Sculptors of African women's bodies: forces reshaping the embodiment of female genital cutting in the west. Arch Sex Behav. 2020. https://doi.org/10.1007/s10508020-01710-1.

23. Poma PA. Race/ethnicity concordance between patients and physicians. J Natl Med Assoc. 2017;109:6-8. https://doi.org/10.1016/j. jnma.2016.12.002.

24. Garces LM, Mickey-Pabello D. Racial diversity in the medical profession: the impact of affirmative action bans on underrepresented student of color matriculation in medical schools. J Furth High Educ. 2015. https://doi.org/10.1353/jhe.2015.0009.

25. University of Arizona College of Medicine - Phoenix. Office of Equity, Diversity and Inclusion. https://phoenixmed.arizona.edu/ diversity. Accessed October 26, 2020.

26. Kennedy BR, Mathis CC, Woods AK. African Americans and their distrust of the health care system: healthcare for diverse populations. J Cult Divers. 2007;14(2):56-60.

27. George S, Duran N, Norris K. A systematic review of barriers and facilitators to minority research participation among African Americans, Latinos, Asian Americans, and Pacific Islanders. Am J Public Health. 2014;104:e16-31. https://doi.org/10.2105/AJPH. 2013.301706.

28. Muse Q 8 Dangerous Myths About Coronavirus Debunked. Philadelphia Magazine. https://www.phillymag.com/healthcarenews/2020/03/19/coronavirus-myths-debunked/. Published 2020. Accessed April 8, 2020.

29. Hogarth RA. The myth of innate racial differences between white and black people's bodies: lessons from the 1793 yellow fever epidemic in Philadelphia, Pennsylvania. Am J Public Health. 2019;109:1339-41. https://doi.org/10.2105/AJPH.2019.305245.

30. Carter C, Sandford III E. The myth of black immunity racialized disease during the COVID-19 pandemic. The St Louis American.

31. Crouse QS. Crisis and emergency risk communication in a pandemic: a model for building capacity and resilience of minority communities. Health Promot Pract. 2008;9:18S-25S. https://doi.org/10. $1177 / 1524839908324022$.

32. Wieland ML, Njeru JW, Alahdab F, Doubeni CA, Sia IG. Community-engaged approaches for minority recruitment into clinical research: a scoping review of the literature. Mayo Clin Proc. 2020. https://doi.org/10.1016/j.mayop.2020.03.028.

33. Johnson CE, Ali SA, Shipp MPL. Building community-based participatory research partnerships with a Somali refugee community. Am J Prev Med. 2009;37:S230-6. https://doi.org/10.1016/j. amepre.2009.09.036.

34. APM Research Lab. The color of coronavirus: COVID-19 deaths by race and ethnicity in the U.S. May 27, 2020. https://www. apmresearchlab.org/covid/deaths-by-race. Accessed June 1, 2020.

35. COVID-19 Pandemic Response, Laboratory Data Reporting: CARES Act Section 18115.

36. Amuta-Jimenez AO, Jacobs W, Smith G. Health disparities and the heterogeneity of blacks/African Americans in the United States: why should we care? Health Promot Pract. 2019;21:492-5. https://doi.org/10.1177/1524839919895507.

37. Williams DR, Lawrence JA, Davis BA. Racism and health: evidence and needed research. Annu Rev Public Health. 2019;40: 105-25. https://doi.org/10.1146/annurev-publhealth-040218043750 .

38. Healthy People. Social Determinants of Health. HealthyPeople. gov. https://www.hhs.gov/sites/default/files/covid-19-laboratorydata-reporting-guidance.pdf. Published 2020. Accessed October 26, 2020.

Publisher's Note Springer Nature remains neutral with regard to jurisdictional claims in published maps and institutional affiliations. 